\title{
Managing the Google Web 1T 5-gram Data Set
}

\author{
Aminul ISLAM \\ Department of Computer Science, SITE \\ University of Ottawa \\ Ottawa, ON, Canada \\ mdislam@site.uottawa.ca
}

\author{
Diana INKPEN \\ Department of Computer Science, SITE \\ University of Ottawa \\ Ottawa, ON, Canada \\ diana@site.uottawa.ca
}

\begin{abstract}
:
This paper describes how the Google Web 1T 5gram data set, contributed by Google Inc., can be stored so that it can be used efficiently with respect to time. We present an efficient way of accessing all the 5-grams for a specific word of interest from the stored files. We measure the maximum access and processing efficiency achievable for any word of interest. We also compare results (access time and memory requirements) on the task of accessing all the 5-grams for a list of words, on both the processed and the original organization of the data set.
\end{abstract}

\section{Keywords:}

Google web 1T; n-gram; 5-grams

\section{Introduction}

The Google Web 1T data set [1], contributed by Google Inc., contains English word $n$-grams (from unigrams to 5grams) and their observed frequency counts calculated over 1 trillion words from web page text collected by Google in January $2006^{1}$. It is expected that this data will be useful for a variety of Research and Development projects, such as statistical machine translation, speech recognition, spelling correction, entity detection, information extraction, as well as for other uses. It contains so much data that many machines with average amounts of memory are unable to even load it. We propose a method for splitting the enormous list of 5-grams, released in 118 large files, down to a more manageable size based on user requirements.

We use the 5-grams from the Web 1T corpus such that the middle token is the term and the two tokens on either side form the context. Several researchers tried to find out the advantages of this context definition by initiating the practical question of what minimum value of window size would, at least in a tolerable fraction of cases, lead to the correct choice of meaning for the central word [2]. A well-known early experiment by Kaplan [3] attempted to answer this question at least in part, by presenting ambiguous words in their original context and in a variant context providing one or two words on either side to seven translators. Kaplan [3] observed that sense resolution given two words on either side

${ }^{1}$ Details of the Google Web 1T can be found at www.ldc.upenn.edu/Catalog/docs/LDC2006T13/readme.txt

978-1-4244-4538-7/09/\$25.00 (C)2009 IEEE. of the word was not significantly better or worse than when given the entire sentence [4]. The same phenomenon has been reported by several researchers: e.g., [5] on Russian, and [6] on French. Whenever we talk about window size, we consider the word of interest in the middle of the window and context words on either side of the word of interest. But we can not use the Web $1 \mathrm{~T} 5$-grams as a window of 5 as all the 5-gram files (total 118 files) are sorted based on the first word in the 5-grams and then the second word in the 5 -grams and so on.

In this article, we wish to find out what maximum $n$-gram access and processing efficiency can be achieved for any word of interest. Searching in the original Web $1 \mathrm{~T}$ dataset is dependent on the total number of $n$-grams of all the words, including the word of interest. Our goal is to make this search independent of the total number of $n$-grams of all the words; this ensures the maximum access and processing efficiency achievable for any word of interest.

Two of our primary assumptions (used in Section 2) are that we need to store less than or equal to $n$ (for our experiment, $n=100,000) 5$-grams in each single file and we want to read only a single file to process all the 5 -grams for a specific word of interest. In Section 3, we omit the first assumption and add a new assumption.

This paper is organized as follow: Section 2 shows how the Web 1T 5-gram data set can be used as a balanced window and how this data can be stored, accessed or processed efficiently with respect to time and memory space and we present experimental results. In Section 3, we find out what maximum $n$-gram access and processing efficiency can be achieved. We summarize contributions in Section 4.

\section{Processing the Web 1T 5-grams}

As the Web 1T 5-grams are sorted based on the first words in the 5-grams, we need to search the middle words in 118 large files of 5-grams. That is, to find the context words of a single word, we need to search all the 5-grams $(1,176,470,663)$, which is not time efficient at all, especially when we are in need of finding context words of a specific word of interest on the fly. So, we process all the Web $1 \mathrm{~T}$ 5 -grams as described in the next sections, in order to make the procedure of finding context words time efficient.

We replace all the times, dates, numbers and the words that start with special characters or numbers with blank spaces in all the 5-grams. If the middle word of a 5-gram is eliminated then we eliminate the whole 5 -gram. We also transform all the 5 -grams into lower case; this creates many duplicate 5-grams (5-grams having the same words). We 


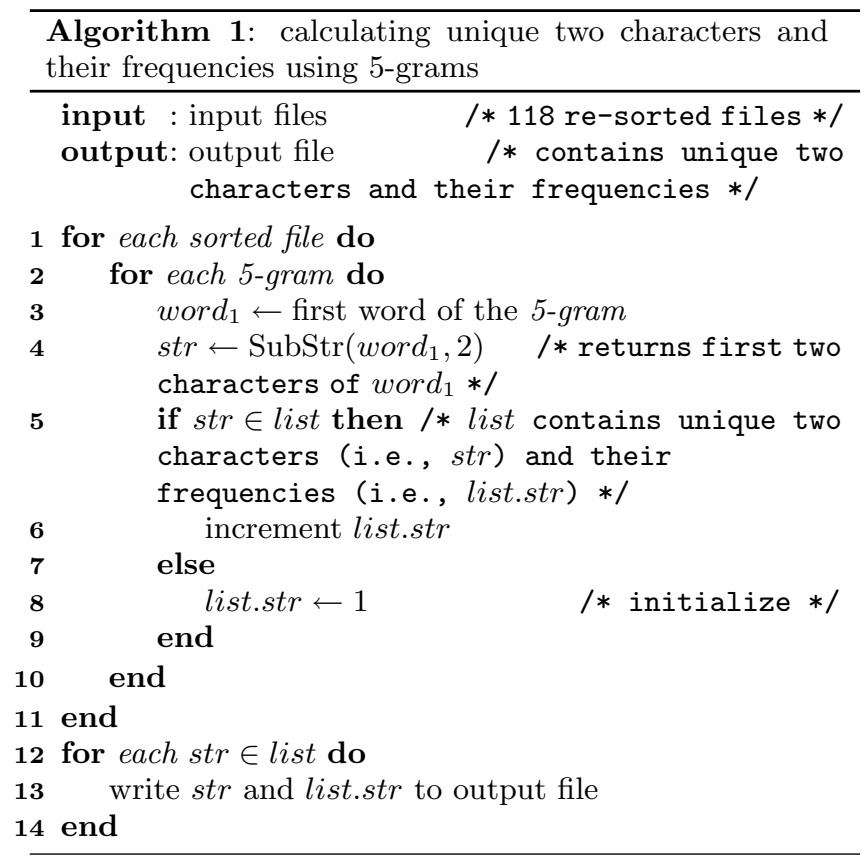

merge all the duplicate 5-grams by adding their frequencies. In this way we managed to reduce the number of 5 -grams from $1,176,470,663$ to $356,611,338$ and the file size of all the 118 files from 33.68 GB to 9.48 GB. Using the same technique, we managed to reduce the number of unigrams from $13,588,391$ to $2,870,385$. We re-sort all the 118 preprocessed 5 -gram files based on the middle words and restore the sorted 5 -gram files to place the middle words in the first position.

\subsection{Calculating Unique Two Characters and Their Fre- quencies using 5-grams}

We devise an algorithm (Algorithm 1) that generates all the unique two-character tokens/words (taking the first two characters from the first word in each 5-gram) and their frequencies from all the 5-grams of 118 re-sorted files.

Now we need to make a decision of what maximum number of 5 -grams ${ }^{2}$ (say $N$ ) we will store in a single file so that we can have efficient search results to find context words for a specific word of interest. Based on the value of $N$, we split the output file generated by Algorithm 1 into two files. One file (say filesName) contains only the words (not frequencies) having frequencies less than $N$. Another file (say nextInFile) also contains only the words (not frequencies) having frequencies greater than or equal to $N$.

\subsection{Calculating Unique $p+1$ Characters and Their Fre- quencies using 5-grams}

We execute Algorithm 2 with nextInFile and $p=2(p$ denotes the number of characters). Algorithm 2 generates all the unique $(p+1)$-character tokens and their frequencies from those 5 -grams of 118 re-sorted files having the first two characters of the first words in nextInFile.

We split the output file generated by Algorithm 2 and add the words having frequencies less than $N$ into the file

${ }^{2}$ For the Web 1T 5-gram we use $N=100,000$ as the stateof-the-art computing device can process these numbers of 5 -grams in less than a second.

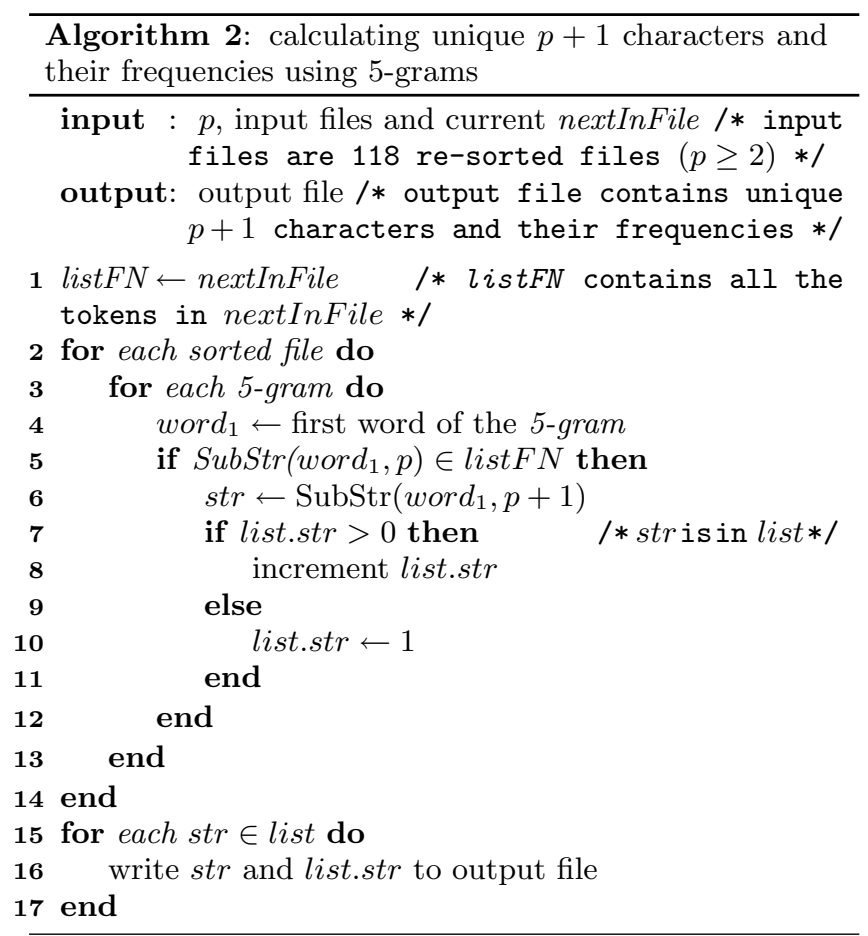

filesName and we store words having frequencies greater than or equal to $N$ by overwriting the file nextInFile. We carry on executing Algorithm 2 with current nextInFile and incremented $p$ (i.e., this time $p=3$ ).

We follow the same steps of executing Algorithm 2 until nextInFile is empty. For the Web $1 \mathrm{~T} 5$-gram data set, we get an empty nextInFile when $p=12$. Finally filesName contains all the file names that need to be created ${ }^{3}$.

\subsection{Creating Files and Assigning 5-grams to Files}

We execute Algorithm 3 which practically creates all the files needed based on filesName entries and assigns each 5gram to the appropriate file (the entries in filesName are the name of the files) traversing all 118 re-sorted 5-gram files.

\subsection{Efficient Search in the Web 1T 5-grams}

To find all the 5-grams for a specific word of interest, we need to search only in a single file among those 22,743 files created using Algorithm 3 in Section 2.3. Algorithm 4 is used to locate the specific file and then to access all the 5grams for that word to further process as per requirement ${ }^{4}$.

\subsection{Comparing Efficiency in Time and Memory Space}

We use [7] data set, a well known data set used to judge different natural language processing (NLP) tasks, to analyze the access time and main memory requirements on run time and to process a specific task ${ }^{5}$. Though there are 30

${ }^{3}$ files Name contains 22,743 entries after running Algorithm 2 on the Web $1 \mathrm{~T} 5$-grams.

${ }^{4}$ We can insert user defined code in line 16 of Algorithm 4.

${ }^{5}$ As a specific task for processing, we access all the 5 -grams of a word of interest and then from those 5-grams we find all the unique context/neighbor words and the pair frequencies. By pair we mean the word of interest and one context/neighbor word. 
Table 1: Experimental Results on Miller and Charles Data Set

\begin{tabular}{|c|c|c|c|c|c|c|c|c|c|c|c|}
\hline \multirow[t]{4}{*}{$\begin{array}{l}\text { Word } \\
\text { Num- } \\
\text { ber }\end{array}$} & \multirow[t]{4}{*}{ Word } & \multicolumn{3}{|c|}{$\begin{array}{l}\text { Time to access all the } 5 \text { - } \\
\text { grams (in seconds) }\end{array}$} & \multicolumn{3}{|c|}{$\begin{array}{l}\text { Time to access all the 5- } \\
\text { grams \& to find all the } \\
\text { context words and the pair } \\
\text { frequencies (in seconds) }\end{array}$} & \multicolumn{2}{|c|}{$\begin{array}{ll}\begin{array}{l}\text { Number } \\
\text { searched }\end{array} & \text { of } \\
\text { grams } & \end{array}$} & \multirow[t]{4}{*}{$\begin{array}{l}\text { Number } \\
\text { of target } \\
5 \text {-grams }\end{array}$} & \multirow[t]{4}{*}{$\begin{array}{l}\text { Memory } \\
\text { required } \\
\text { to run (in } \\
\text { bytes) }\end{array}$} \\
\hline & & \multirow{2}{*}{\multicolumn{2}{|c|}{$\begin{array}{l}\text { Dual Core } \\
\text { Machine }\end{array}$}} & \multirow{3}{*}{$\begin{array}{l}\text { Think } \\
\text { Centre } \\
\text { (TER } \\
0.67)\end{array}$} & \multirow{2}{*}{\multicolumn{2}{|c|}{$\begin{array}{l}\text { Dual Core } \\
\text { Machine }\end{array}$}} & \multirow{3}{*}{$\begin{array}{l}\text { Think } \\
\text { Centre } \\
\text { (TER } \\
0.67)\end{array}$} & \multirow{3}{*}{$\begin{array}{l}\text { TER } \\
0.67\end{array}$} & \multirow[t]{3}{*}{ TER 1} & & \\
\hline & & & & & & & & & & & \\
\hline & & $\begin{array}{l}\text { TER } \\
0.67\end{array}$ & $\begin{array}{l}\text { TER } \\
1\end{array}$ & & $\begin{array}{l}\text { TER } \\
0.67\end{array}$ & $\begin{array}{l}\text { TER } \\
1\end{array}$ & & & & & \\
\hline 1 & car & 1.66 & 0.07 & 3.39 & 4.35 & 3.65 & 5.5 & 272,920 & 272,532 & 272,532 & 455,583 \\
\hline 2 & automobile & 0.28 & 0.01 & 0.58 & 0.42 & 0.18 & 0.69 & 47,395 & 11,863 & 11,863 & 57,993 \\
\hline 3 & gem & 0.19 & 0.00 & 0.38 & 0.30 & 0.14 & 0.47 & 31,891 & 9,342 & 9,342 & $\begin{array}{l}58,488 \\
\end{array}$ \\
\hline 4 & jewel & 0.40 & 0.00 & 0.11 & 0.13 & 0.12 & 0.19 & 8,263 & 7,936 & 7,936 & 53,606 \\
\hline 5 & journey & 0.13 & 0.01 & 0.27 & 0.33 & 0.27 & 0.45 & 23,996 & 19,508 & 19,508 & 93,099 \\
\hline 6 & voyage & 0.37 & 0.00 & 0.76 & 0.46 & 0.07 & 0.80 & 60,527 & 4,684 & 4,684 & 37,016 \\
\hline 7 & boy & 0.40 & 0.02 & 0.81 & 1.08 & 0.93 & 1.42 & 67,943 & 67,391 & 67,391 & 209,030 \\
\hline 8 & lad & 0.01 & 0.00 & 0.03 & 0.03 & 0.03 & 0.05 & 2,471 & 1,837 & 1,837 & 18,424 \\
\hline 9 & coast & 0.51 & 0.02 & 1.08 & 1.12 & 0.82 & 1.55 & 86,362 & 59,030 & 59,030 & 170,044 \\
\hline 10 & shore & 0.15 & 0.00 & 0.33 & 0.33 & 0.23 & 0.48 & 27,400 & 15,837 & 15,837 & 78,535 \\
\hline 11 & asylum & 0.09 & 0.00 & 0.17 & 0.15 & 0.07 & 0.23 & 15,656 & 5,057 & 5,057 & 35,086 \\
\hline 12 & madhouse & 0.01 & 0.00 & 0.03 & 0.02 & 0.00 & 0.03 & 2,123 & 179 & 179 & 3,057 \\
\hline 13 & magician & 0.39 & 0.01 & 0.81 & 0.46 & 0.04 & 0.83 & 70,113 & 2,497 & 2,497 & 21,479 \\
\hline 14 & wizard & 0.12 & 0.00 & 0.26 & 0.28 & 0.21 & 0.39 & 22,324 & 14,471 & 14,471 & 75,424 \\
\hline 15 & midday & 0.54 & 0.00 & 1.11 & 0.61 & 0.01 & 1.11 & 91,534 & 992 & 992 & 10,069 \\
\hline 16 & noon & 0.10 & 0.00 & 0.20 & 0.17 & 0.08 & 0.27 & 18,058 & 5,806 & 5,806 & 31,747 \\
\hline 17 & furnace & 0.03 & 0.00 & 0.06 & 0.06 & 0.05 & 0.09 & 4,952 & 3,104 & 3,104 & 23,627 \\
\hline 18 & stove & 0.05 & 0.00 & 0.11 & 0.10 & 0.07 & 0.14 & 8,340 & 4,747 & 4,747 & 28,568 \\
\hline 19 & food & 0.97 & 0.05 & 2.05 & 2.68 & 2.28 & 3.44 & 171,215 & 170,079 & 170,079 & 305,285 \\
\hline 20 & fruit & 0.32 & 0.01 & 0.66 & 0.63 & 0.39 & 0.91 & 57,135 & 28,403 & 28,403 & 108,827 \\
\hline 21 & bird & 0.40 & 0.01 & 0.86 & 0.85 & 0.59 & 1.20 & 71,947 & 40,779 & 40,779 & 152,996 \\
\hline 22 & cock & 0.39 & 0.02 & 0.77 & 0.97 & 0.83 & 1.22 & 60,695 & 60,424 & 60,424 & 92,293 \\
\hline 23 & tool & 0.50 & 0.02 & 1.06 & 1.40 & 1.18 & 1.78 & 87,837 & 87,022 & 87,022 & 222,354 \\
\hline 24 & implement & 0.14 & 0.01 & 0.28 & 0.41 & 0.34 & 0.53 & 26,026 & 26,022 & 26,022 & 82,762 \\
\hline 25 & brother & 0.35 & 0.01 & 0.73 & 0.72 & 0.46 & 1.03 & 63,185 & 34,244 & 34,244 & 119,188 \\
\hline 26 & monk & 0.13 & 0.00 & 0.28 & 0.19 & 0.06 & 0.33 & 24,507 & 3,630 & 3,630 & 32,736 \\
\hline 27 & oracle & 0.12 & 0.01 & 0.27 & 0.32 & 0.27 & 0.44 & 21,346 & 19,624 & 19,624 & 84,541 \\
\hline 28 & rooster & 0.05 & 0.00 & 0.09 & 0.08 & 0.03 & 0.11 & 8,580 & 1,924 & 1,924 & 18,374 \\
\hline 29 & cemetery & 0.13 & 0.00 & 0.27 & 0.25 & 0.15 & 0.36 & 22,317 & 10,286 & 10,286 & 64,289 \\
\hline 30 & woodland & 0.05 & 0.00 & 0.09 & 0.11 & 0.08 & 0.16 & 9,048 & 5,286 & 5,286 & 39,352 \\
\hline 31 & hill & 0.44 & 0.02 & 0.91 & 1.22 & 1.06 & 1.56 & 74,961 & 73,998 & 73,998 & 251,043 \\
\hline 32 & slave & 0.55 & 0.01 & 1.14 & 0.77 & 0.24 & 1.28 & 98,079 & 16,573 & 16,573 & 72,514 \\
\hline 33 & forest & 0.42 & 0.01 & 0.88 & 0.95 & 0.69 & 1.31 & 73,224 & 48,829 & 48,829 & 174,405 \\
\hline 34 & graveyard & 0.17 & 0.00 & 0.38 & 0.21 & 0.03 & 0.39 & 32,559 & 1,262 & 1,262 & 15,320 \\
\hline 35 & chord & 0.14 & 0.00 & 0.28 & 0.19 & 0.05 & 0.30 & 24,158 & 3,384 & 3,384 & 23,465 \\
\hline 36 & smile & 0.54 & 0.00 & 1.14 & 0.71 & 0.14 & 1.22 & 98,610 & 10,149 & 10,149 & 56,234 \\
\hline 37 & glass & 0.49 & 0.03 & 1.03 & 1.36 & 1.15 & 1.72 & 84,160 & 83,244 & 83,244 & 206,989 \\
\hline 38 & string & 0.47 & 0.02 & 0.98 & 1.16 & 0.90 & 1.56 & 87,937 & 66,204 & 66,204 & 236,570 \\
\hline 39 & crane & 0.13 & 0.00 & 0.28 & 0.20 & 0.09 & 0.33 & 23,252 & 5,425 & 5,425 & 43,547 \\
\hline
\end{tabular}




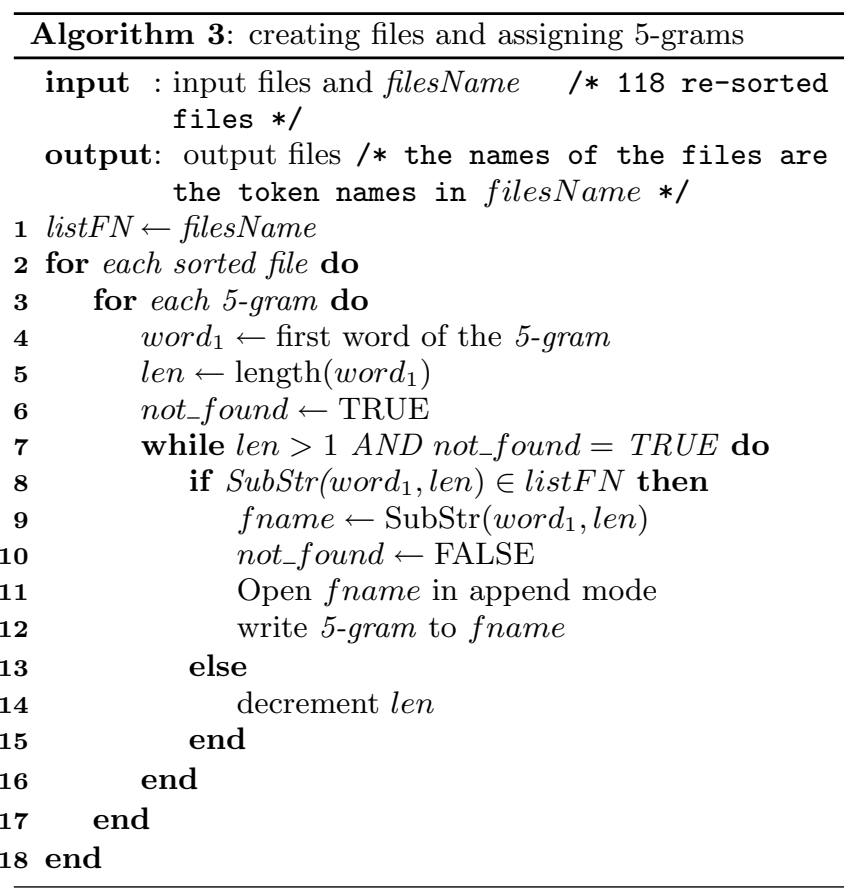

pairs of words in this data set, the number of distinct words are 39. We experimented on two different machines. The first machine is a dual core Intel $\AA \mathrm{Xeon}^{\mathrm{TM}}$ having $\mathrm{CPU}$ speed of $3.20 \mathrm{GHz}$ and main memory of $4 \mathrm{~GB}$ (we refer it as Dual Core Machine). The second machine is an IBM ThinkCentre M51 machine with Intel@ Pentium 4 processor having CPU speed of $3.40 \mathrm{GHz}$ and main memory of $1 \mathrm{~GB}$ (we refer it as ThinkCentre). Table 1 shows all the test results on [7] data set. Table 1 shows that 38 words out of $39(97.4 \%)$ were accessed in less than a second. The total access time for all the 39 words on this machine is 12 seconds, an average of 0.31 seconds per word which is almost half compared to the ThinkCentre (0.64 seconds). The first word, car, takes longer time to be accessed or to be processed because it has more 5-grams than the other words.

Table 1 also shows a comparison between the number of 5 -grams we search through (we call them searched 5-grams) versus the number of 5 -grams we needed (we call them target 5 -grams). The number of searched 5 -grams and the number of target 5-grams are independent of the computing devices. The ratio between the number of target 5-grams and the number of searched 5-grams (we call it Time Efficiency Ratio $(T E R))$ is a key issue for efficiency in terms of time. The higher the TER the better. Having the time efficiency ratio equal to 1 is an ideal case. The average time efficiency ratio for all the 39 words using the original 118 files is 0.001108 (this is the base case). The average time efficiency ratio for all the 39 words using 22,743 files is 0.63 . To have an ideal average time efficiency ratio, we need to have a distinct 5-gram file for each unique word (unigram) possible. In Section 3, we study the feasibility and practicability of generating this enormous number of files.

\section{Making the time efficiency ratio (TER) equal to 1}

To have an ideal TER, we use a new assumption (instead of the first assumption used in Section 2) that we store only

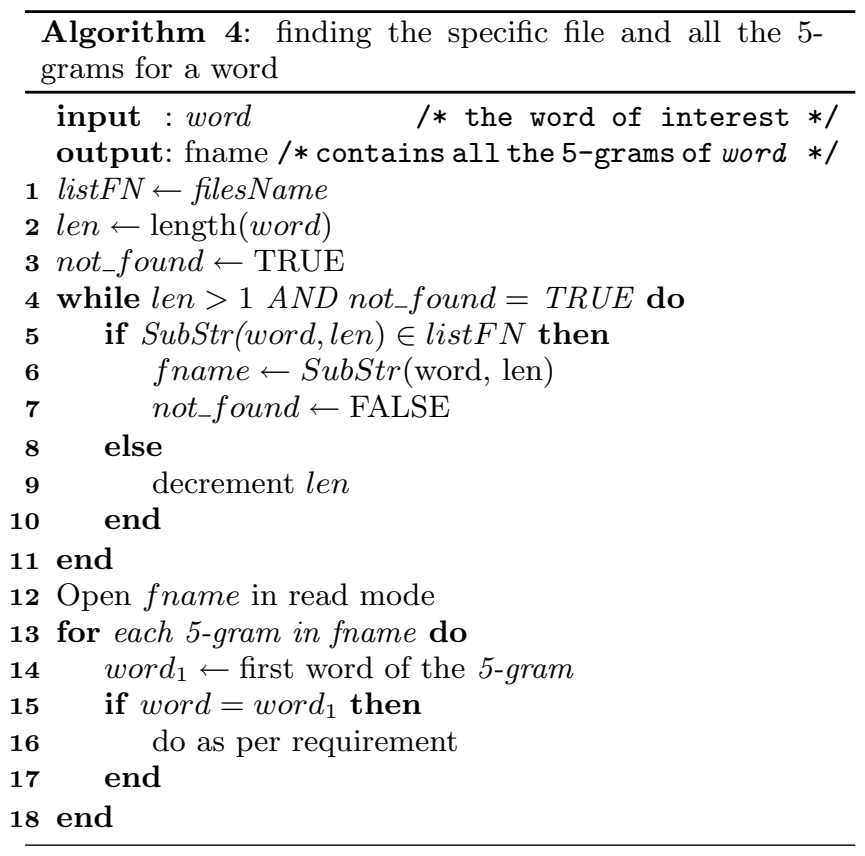

all the 5-grams of each unique word in a single file. We also use the second assumption used in Section 2.

\subsection{Creating Files and Assigning 5-grams when TER=1}

We execute Algorithm 5 which creates unique files to store all the 5-grams associated with each unique words and assigns each 5-gram to the appropriate file traversing all 118 re-sorted 5-gram files. Algorithm 5 generates 2,870,385 files from the 118 re-sorted files and all these files are stored in a subfolder $s f\left(s f \in\left\{\{a \cdots z\} \times\left\{0 \cdots 9,{ }^{\prime}, a \cdots z\right\}\right\}\right)$ under the folder $f(f \in\{a \cdots z\})$. So, there will have $26 \times 37=962$ unique subfolders under 26 folders. For example, all the 5grams of word brother are in subfolder $b r$ under folder $b$ (the path of the file is /home/.../b/br/brother $)$.

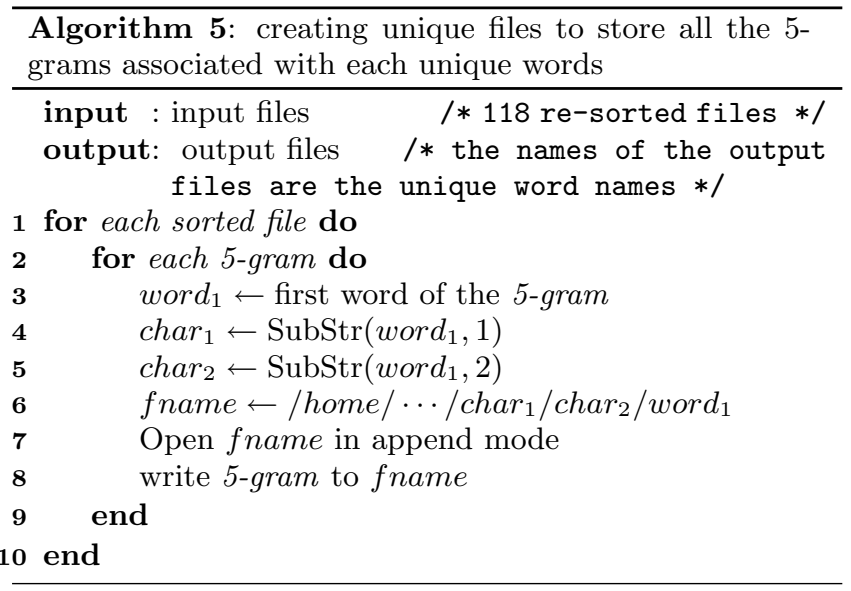

\subsection{Efficient Search when TER $=1$}

To find all the 5-grams for a specific word of interest, we need to access ${ }^{6}$ only a single file among those 2,870,385 files, 


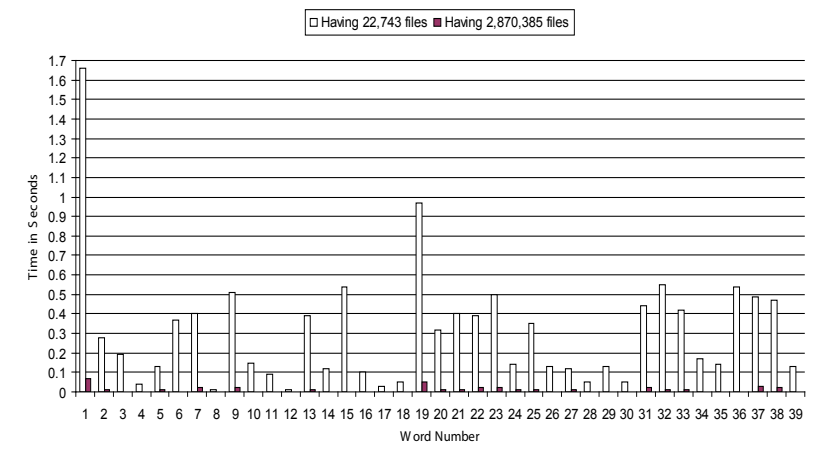

Figure 1: Comparison between time (in seconds) needed to access all the 5-grams of the 39 words on the Dual Core Machine having 22,743 files versus having $2,870,385$ files.

distributed under 962 different subfolders, created using Algorithm 5 in Section 3.1. Algorithm 6 is used to locate the specific file that has all the 5-grams for a specific word and then to access all the 5-grams for that word to further process as per requirement ${ }^{7}$.

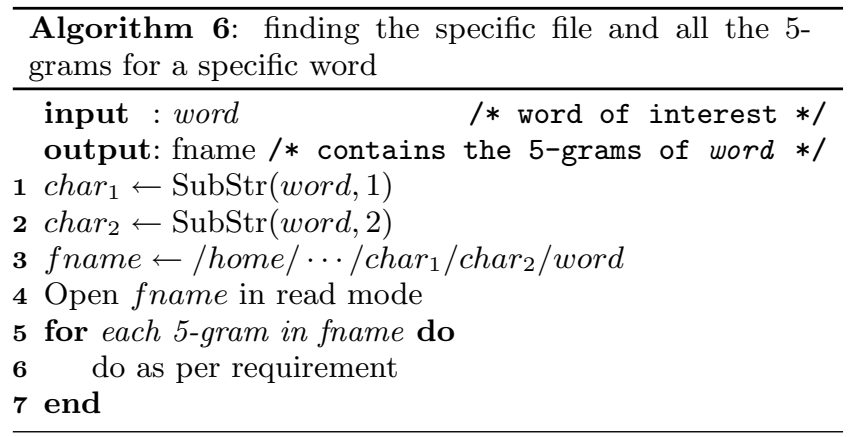

\subsection{Comparing Efficiency when TER $=1$}

We use the same [7] data set on the Dual Core Machine. Table 1 shows all the test results on [7] data set when TER = 1. Table 1 shows that all the 39 words $(100 \%)$ were accessed in less than 0.1 seconds using 2,870,385 files. The total access time for all the 39 words on the Dual Core Machine is 0.37 seconds, an average of 0.0095 seconds per word (i.e., $96.95 \%$ less average access time compared to using 22,743 files). Figure 1 shows a comparison between the time (in seconds) needed to access all the 5 -grams of the 39 words on the Dual Core Machine using the 22,743 files from Section 2 versus using $2,870,385$ files $^{8}$. The single largest file with respect to the memory size is new having $1,310,730$-grams and using $32,338,060$ bytes (32.34 MB) of memory when we use $2,870,385$ files. So, the maximum main memory required for any word is $32.34 \mathrm{MB}$. It takes 0.39 seconds on the Dual Core Machine to access all the 5 -grams and 17.12 seconds $^{9}$ to access all the 5 -grams and then to find all the context file are target 5-grams.

${ }^{7}$ We can insert code for what we want to do with these 5grams in line 6 of Algorithm 6.

${ }^{8}$ There are 20 words in Figure 1 having 0.00 second access time as the Benchmark module of Perl returns only 2 digits after decimal point.

${ }^{9}$ We need $95.44 \%$ less time to only access all the 5-grams
Table 2: File Size on Disk

\begin{tabular}{|l|l|l|l|}
\hline $\begin{array}{l}\text { No. of } \\
\text { files }\end{array}$ & $\begin{array}{l}\text { No. of } \\
\text { folders }\end{array}$ & $\begin{array}{l}\text { Actual size (in } \\
\text { bytes) }\end{array}$ & $\begin{array}{l}\text { Size on disk } \\
\text { (in bytes) }\end{array}$ \\
\hline 118 & N/A & $9,483,356,102$ & $9,483,418,624$ \\
\hline 22,743 & N/A & $9,483,356,102$ & $9,495,010,304$ \\
\hline $2,870,385$ & 988 & $6,865,896,655$ & $9,514,983,424$ \\
\hline
\end{tabular}

words and the pair frequencies for the word new. We can consider these values as the upper limits. Table 2 shows the actual size of the files and the size required on disk for different number of files ${ }^{10}$.

\section{Conclusions}

It is expected that Google Inc. will release a larger data set than the Web $1 \mathrm{~T}$ in the future and our proposed method is general enough to handle a larger data set than the Web 1T. Assuming that the rate of increase of the number of $n$ grams is much smaller than the rate of increase of the number of words (collected from web pages) over which their observed frequency are counted, our proposed approach is easily applicable to the future Web $10 \mathrm{~T}$, Web $100 \mathrm{~T}$ or even Web $1 \mathrm{Q}(\mathrm{Q}=$ Quadrillion). We find that if the computing device supports millions of files, then creating unique files to store all the 5-grams associated with each unique word provides the best access time efficiency and processing efficiency. Otherwise, the method described in Section 2 provides a reasonable access time efficiency and processing efficiency. It is interesting to note that though we only experimented on the 5 -gram data set, the proposed methods are also applicable to Web $1 \mathrm{~T}$ bigrams, trigrams, and 4-grams.

\section{References}

[1] T. Brants and A. Franz, "Web 1T 5-gram corpus version 1.1.," tech. rep., Google Research, 2006.

[2] W. Weaver, Translation. 1949. Reprinted in Locke, William N. and Booth, A. Donald (1955) (Eds.), Machine translation of languages, John Wiley \& Sons, New York, 15-23.

[3] A. Kaplan, An experimental study of ambiguity and context. November 1950. Published as Kaplan, Abraham (1955), An experimental study of ambiguity and context, Mechanical Translation, 2(2), 39-46.

[4] N. Ide and J. Véronis, "Word sense disambiguation: The state of the art," Computational Linguistics, vol. 24, no. 1, pp. 1-41, 1998.

[5] A. K. Koutsoudas and R. Korfhage, "M.T. and the problem of multiple meaning," Mechanical Translation, vol. 2, no. 2, pp. 46-51, 1956.

[6] Y. Choueka and S. Lusignan, "Disambiguation by short contexts," Computers and the Humanities, vol. 19, pp. 147-158, 1985.

[7] G. A. Miller and W. G. Charles, "Contextual correlates of semantic similarity," Language and Cognitive Processes, vol. 6, no. 1, pp. 1-28, 1991.

and $7.06 \%$ less time to access all the 5-grams and then to find all the context words and the pair frequencies for the word new compared to using 22,743 files.

${ }^{10}$ The actual file size using $2,870,385$ files is less than the other two versions because we do not need to store the word of interest as the file name itself represents it. 